\title{
Radical Grafting from Carbon Black. Graft Polymerization of Vinyl Monomers Initiated by Azo Groups Introduced onto Carbon Black Surface
}

\author{
Kazuhiro FujIKI, Norio Tsubokawa, ${ }^{*}$ and Yasuo SonE* \\ Graduate School of Science and Technology, Niigata University, 8050, \\ Ikarashi 2-nocho, Niigata 950-21, Japan \\ *Department of Material and Chemical Engineering, Faculty of Engineering, \\ Niigata University, 8050, Ikarashi 2-nocho, Niigata 950-21, Japan
}

(Received January 19, 1990)

\begin{abstract}
The radical graft polymerization of vinyl monomers from carbon black initiated by azo groups introduced onto the surface was investigated. The introduction of azo groups onto the carbon black surface was achieved by three methods: (1) the reaction of 2,2'-azobis(2-cyano$n$-propanol) with acyl chloride groups on the surface, which were introduced by the reaction of carboxyl groups on the surface with thionyl chloride, (2) the reaction of 4,4'-azobis(4-cyanovaleric acid) with isocyanate groups on the surface, which were introduced by the treatment of carbon black with tolylene 2,4-diisocyanate, and (3) the reaction of hydroxyl groups on the surface with $4,4^{\prime}$-azobis(4-cyanovaleryl chloride). It was found that the polymerization of vinyl monomers such as methyl methacrylate and styrene was initiated by azo groups introduced onto the carbon black surface. During the polymerization, the corresponding polymer was effectively grafted onto the carbon black surface based on the propagation of the polymer from the radical produced on the surface by the decomposition of the azo groups. The percentage of grafting of poly(methyl methacrylate) and polystyrene using azo groups introduced by the method 2 increased to $40.0 \%$ and $28.5 \%$, respectively. Furthermore, the effects of temperature and $e$-value of vinyl monomers on the graft polymerization were discussed.

KEY WORDS Carbon Black / Graft Polymerization / Polymer-Grafted Carbon Black / Azo Groups / $e$-Value / 4,4'-Azobis(4-cyanovaleric acid) / Vinyl Monomer / 2,2'-Azobis(2-cyano- $n$-propanol) /
\end{abstract}

The dispersibility of carbon black in a polymer matrix or an organic solvent is remarkably improved by the grafting of polymers onto the surface. This is due to that the grafted polymer chains on carbon black interfere with the aggregation of the carbon black particles and increase the affinity of the surface for solvents. Therefore, the dispersibility of the polymer-grafted carbon black increases with an increase in the percentage of grafting. ${ }^{1,2}$

Although a polymer is grafted onto the surface during the polymerization in the presence of carbon black using an initiator, the percentage of grafting is very low. To prepare the polymer-grafted carbon black with a higher percentage of grafting, it is necessary to initiate the graft polymerization from active sites on the carbon black surface. ${ }^{3,4}$

In a series of our papers, we reported the grafting of various kinds of polymers onto carbon black by the anionic grafting from alkali metal carboxylate groups on carbon black $^{5,6}$ and by the cationic grafting from acylium perchlorate groups on carbon black. ${ }^{7}$ The radical grafting from carbon black, however, has been scarcely investigated.

In the previous paper, we reported the radical graft polymerization of vinyl monomers from carbon black initiated by a redox system 
consisting of ceric ions and alcoholic hydroxyl groups introduced onto the surface. ${ }^{8}$ Furthermore, we succeeded in the radical graft polymerization of vinyl monomers initiated by peroxyester groups introduced onto the surface. ${ }^{9}$ In this polymerization, vinyl polymers with positive $e$-value were effectively grafted onto the carbon black surface based on the propagation of polymers from the radicals produced on the surface. The radical graft polymerization of vinyl monomers with negative $e$-value, however, failed to initiate by peroxyester groups on the surface. ${ }^{9}$

In the present paper, the introduction of azo groups onto the surface of the carbon black and the graft polymerization of vinyl monomers initiated by the azo groups on the surface were investigated (eq 1). Furthermore, the effect of $e$-value of vinyl monomers on the graft polymerization was discussed.

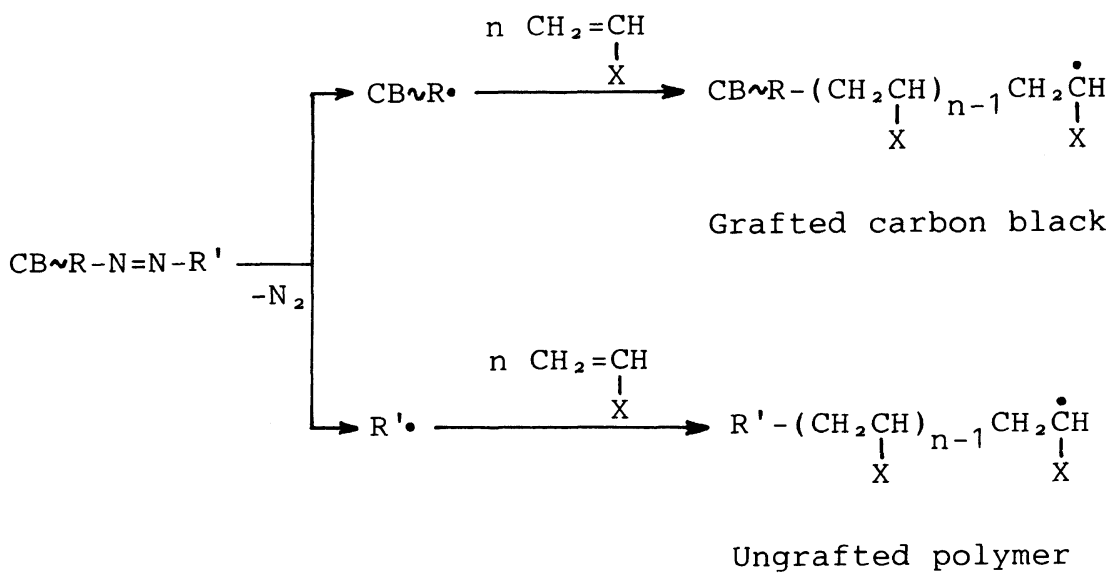

\section{EXPERIMENTAL}

\section{Materials}

The carbon black used was color channel black Neospectra II (Columbian Carbon Co., BET specific surface area, $906 \mathrm{~m}^{2} \mathrm{~g}^{-1}$ ), dried in vacuo at $110^{\circ} \mathrm{C}$ before use. The content of carboxyl, phenolic hydroxyl, and quinonic oxygen groups on the surface was determined to be $0.40,0.24$, and $0.92 \mathrm{mmol} \mathrm{g}^{-1}$, respectively.

Methyl methacrylate (MMA), styrene, and acrylonitrile were purified by general methods and distilled twice. $N$-Vinyl-2-pyrrolidone, glycidyl methacrylate, 4-vinylpyridine, acrylic acid, and 2-hydroxyethyl methacrylate were distilled under reduced pressure in the presence of hydroquinone. Vinyl acetate and methyl vinyl ketone were distilled twice.

Tolylene 2,4-diisocyanate (TDI) obtained from Wako Pure Chemical Co., Ltd. was distilled under reduced pressure just before its use. 4,4'-Azobis(4-cyanovaleric acid) (ACV) obtained from Otsuka Chemical Co., Ltd. and 2,2'-azobis(2-cyano-n-propanol) (ACP) obtained from Wako Pure Chemical Co., Ltd. were dried in vacuo at room temperature before use.

4,4'-Azobis(4-cyanovaleryl chloride) (ACV$\mathrm{COCl})$ was prepared by the reaction of $\mathrm{ACV}$ with thionyl chloride according to a method of literature. ${ }^{10}$

Dioxane and tetrahydrofuran (THF) were refluxed over sodium and distilled twice. Dimethyl sulfoxide (DMSO) was dried over calcium hydride with stirring for two days and then distilled twice under reduced pressure. Other solvents and reagents were used without further purification. 
Introduction of Acyl Chloride Groups onto Carbon Black

Carbon black having acyl chloride groups was prepared by the reaction of carboxyl groups on the surface with thionyl chloride. The procedures were described in detail in the previous paper. ${ }^{7,11}$

\section{Introduction of Hydroxyl Groups onto Carbon Black}

The introduction of hydroxyl groups onto the carbon black surface was carried out by the reaction of carboxyl, phenolic hydroxyl, and quinonic oxygen groups on the surface with $n$-butyllithium (BuLi). ${ }^{12}$ The procedures were as follows. Into a $200 \mathrm{ml}$ flask, $6.0 \mathrm{~g}$ of carbon black, $10.0 \mathrm{ml}$ of BuLi (about 15\% hexane solution), $60.0 \mathrm{ml}$ of THF were charged. The mixture was stirred with a magnetic stirrer for $2 \mathrm{~h}$ at $25^{\circ} \mathrm{C}$ under dry nitrogen. After the reaction, the resulting carbon black was filtered, washed with distilled water until the filtrate was neutral, and dried in vacuo at $110^{\circ} \mathrm{C}$. The treated carbon black was stored in a desiccator at room temperature.

\section{Introduction of Azo Groups onto Carbon Black}

The introduction of azo groups onto the surface was achieved by the following three methods.

(1) Preparation of Carbon Black 1: Carbon black 1 was prepared by the reaction of acyl chloride groups on the surface with ACP. Into a $200 \mathrm{ml}$ flask, $6.0 \mathrm{~g}$ of carbon black having acyl chloride groups, $1.0 \mathrm{~g}$ of ACP, $80.0 \mathrm{ml}$ of dioxane, and $\alpha$-picoline were charged. Then the reaction mixture was stirred with a magnetic stirrer for $8 \mathrm{~h}$ at $25^{\circ} \mathrm{C}$ under dry nitrogen. After the reaction, the resulting carbon black was repeatedly washed with methanol and dried in vacuo at room temperature. The treated carbon black was stored in the dark below $0^{\circ} \mathrm{C}$.

(2) Preparation of Carbon Black 2: Carbon black 2 was prepared by the reaction of isocyanate groups on the surface with ACV. A typical example was as follows. Into a $200 \mathrm{ml}$ flask, $5.0 \mathrm{~g}$ of carbon black, $130 \mathrm{ml}$ of DMSO, $1.0 \mathrm{ml}$ of TDI, and $\alpha$-picoline were charged..$^{13,14}$ The mixture was stirred with a magnetic stirrer at $60^{\circ} \mathrm{C}$ under dry nitrogen, and after the reaction for $4 \mathrm{~h}$, the flask was cooled to room temperature. Then $4.0 \mathrm{~g}$ of $\mathrm{ACV}$ was added into the flask and the reaction was continued with stirring for $8 \mathrm{~h}$ at $25^{\circ} \mathrm{C}$. Subsequent procedures of washing and drying of the resulting carbon black were performed in the same manner as described above.

(3) Preparation of Carbon Black 3: Carbon black 3 was prepared by the reaction of hydroxyl groups on the surface with ACV$\mathrm{COCl}$. A typical example was as follows. Into a $200 \mathrm{ml}$ flask, $6.0 \mathrm{~g}$ of carbon black, $0.6 \mathrm{~g}$ of $\mathrm{ACV}-\mathrm{COCl}, 80.0 \mathrm{ml}$ of dioxane, and $\alpha$-picoline were charged. When the BuLi-treated carbon black was used, $4.9 \mathrm{~g}$ of $\mathrm{ACV}-\mathrm{COCl}$ was reacted. Then the reaction mixture was stirred with a magnetic stirrer for $8 \mathrm{~h}$ at $25^{\circ} \mathrm{C}$ under dry nitrogen. Subsequent procedures of washing and drying were performed in the same manner as described above.

\section{Polymerization Procedures}

Into a glass tube, $0.30 \mathrm{~g}$ of carbon black and $10.0 \mathrm{ml}$ of monomer were charged. The tube was cooled, thawed three times with nitrogen, and sealed under vacuum. The sealed tube was heated at $70^{\circ} \mathrm{C}$ with shaking. After a definite time, the contents of the tube were poured into a large excess of precipitant for the polymer. The precipitate was filtered and dried in vacuo at $40^{\circ} \mathrm{C}$.

When $N$-vinyl-2-pyrrolidone and acrylic acid were used as monomer, the polymerization was stopped by adding hydroquinone and then unreacted monomer was removed by distillation under reduced pressure. The residue was dried in vacuo at $40^{\circ} \mathrm{C}$.

The conversion was calculated by the following equation: 
Conversion (\%)

$$
=\frac{\begin{array}{c}
\text { Precipitate obtained }- \text { Carbon black } \\
(\mathrm{g})
\end{array}}{\text { Monomer used }} \times 100
$$

(g)

\section{Percentage of Grafting}

To separate ungrafted polymer from the reaction product that contained carbon black, the product was dispersed in solvent for the polymer and centrifuged at $1.2 \times 10^{4} \mathrm{rpm}$ until the carbon black was precipitated completely. The carbon black precipitated was extracted with solvent for the polymer using a Soxhlet extractor until no more polymer could be detected in the refluxing solvent. The percentage of grafting was determined by the following equation:

$$
\text { Grafting }(\%)=\frac{\text { Polymer grafted }(\mathrm{g})}{\text { Carbon black used }(\mathrm{g})} \times 100
$$

\section{RESULTS AND DISCUSSION}

\section{Introduction of Azo Groups onto Carbon} Black

The introduction of azo groups onto the carbon black surface was achieved by the following three methods: (1) the reaction of acyl chloride groups on the surface, which were introduced by the reaction of carboxyl groups on the surface with thionyl chloride, with ACP (eq 2), (2) the reaction of isocyanate groups on the surface, which were introduced by the treatment of carbon black with TDI, with ACV (eq 3), and (3) the reaction of hydroxyl groups on the surface with $\mathrm{ACV}-\mathrm{COCl}$ (eq 4).

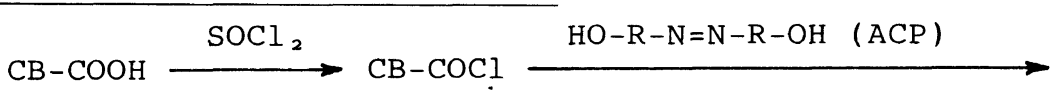

$$
\mathrm{CB}-\mathrm{COO}-\mathrm{R}-\mathrm{N}=\mathrm{N}-\mathrm{R}-\mathrm{OH}
$$

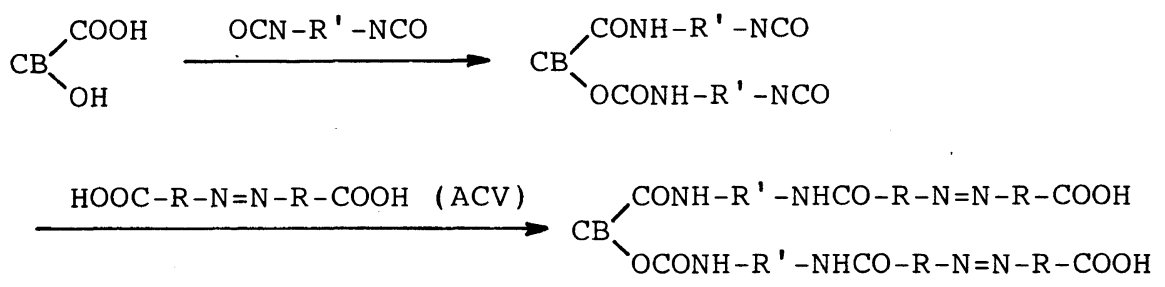

2

$$
\mathrm{CB}-\mathrm{OH} \stackrel{\mathrm{ClOC}-\mathrm{R}-\mathrm{N}=\mathrm{N}-\mathrm{R}-\mathrm{COCl}(\mathrm{ACV}-\mathrm{COCl})}{\longrightarrow} \mathrm{CB}-\mathrm{OCO}-\mathrm{R}-\mathrm{N}=\mathrm{N}-\mathrm{R}-\mathrm{COCl}
$$

The reaction of carbon black with $\mathrm{ACP}$, $\mathrm{ACV}$, or $\mathrm{ACV}-\mathrm{COCl}$ was carried out at $25^{\circ} \mathrm{C}$. Therefore, it is not necessary to take into account for the decomposition of the azo compounds during the reaction, because the half-lives of them are reported to be more than 10000 min. $^{15}$

The amount of azo groups introduced onto the surface of the carbon black was determined by elemental (nitrogen) analysis. The results are 
Table I. The amount of azo groups introduced onto carbon black surface

\begin{tabular}{|c|c|c|c|c|c|}
\hline \multirow{2}{*}{ Carbon black } & $\mathrm{COOH}$ & $\mathrm{OH}$ & \multirow{2}{*}{$\begin{array}{l}\text { Introduction } \\
\text { reaction }\end{array}$} & \multirow{2}{*}{ No. } & \multirow{2}{*}{$\frac{\text { Azo group }}{\mathrm{mmol}^{-1}}$} \\
\hline & $\mathrm{mmol} \mathrm{g}^{-1}$ & $\mathrm{mmol} \mathrm{g}^{-1}$ & & & \\
\hline Neospectra II & 0.40 & 0.24 & - & - & 0 \\
\hline Neospectra II & 0.40 & 0.24 & eq 2 & 1 & 0.18 \\
\hline Neospectra II & 0.40 & 0.24 & eq 3 & 2 & 0.33 \\
\hline Neospectra II & 0.40 & 0.24 & eq 4 & 3 & 0.13 \\
\hline $\begin{array}{l}\text { BuLi-treated } \\
\text { Neospectra II }\end{array}$ & 0 & 1.96 & eq 4 & 4 & 0.48 \\
\hline
\end{tabular}

summarized in Table I.

As shown in Table I, it is apparent that azo groups can be introduced onto the carbon black surface by the reaction according to the methods 1, 2, and 3 (eq 2, 3, and 4). The azo group content of carbon black increased, depending on the introduction reaction, in the following order: $3<1<2$. This order agreed with increasing amount of starting functional groups used for the introduction of azo groups: the functional groups used for the introduction of azo groups by the method 3,1 , and 2 are phenolic hydroxyl groups $\left(0.24 \mathrm{mmol} \mathrm{g}^{-1}\right)$, carboxyl groups $\left(0.40 \mathrm{mmol} \mathrm{g}^{-1}\right)$, and carboxyl and phenolic hydroxyl groups $(0.40+0.24=$ $0.64 \mathrm{mmol} \mathrm{g}^{-1}$ ), respectively.

To increase the azo group content of carbon black, hydroxyl groups were introduced onto carbon black. The introduction of hydroxyl groups was achieved by the hydrolysis of phenoxy and alkoxy lithium groups, which were introduced by the reaction of BuLi with phenolic hydroxyl, carboxyl, and quinonic oxygen groups on the surface (eq 5,6 , and 7). ${ }^{12}$ By the treatment the hydroxyl group content of the carbon black increased to $1.96 \mathrm{mmol} \mathrm{g}^{-1}$. Carbon black 4 was prepared by the reaction of hydroxyl groups on the treated carbon black with ACV-COCl. The amount of azo groups introduced onto carbon black 4 increased to $0.48 \mathrm{mmol} \mathrm{g}^{-1}$ as shown in Table I.

Based on the above results, it is concluded that azo groups can be introduced onto the carbon black surface according to the methods as shown in eqs 2,3 , and 4.

$$
\begin{aligned}
& \mathrm{CB}-\mathrm{OH} \stackrel{\mathrm{BuLi}}{\longrightarrow} \mathrm{CB}-\mathrm{OLi} \stackrel{\mathrm{H}_{2} \mathrm{O}}{\longrightarrow} \mathrm{CB}-\mathrm{OH} \\
& \mathrm{CB}-\mathrm{COOH} \stackrel{2 \mathrm{BuLi}}{\longrightarrow} \mathrm{CB}-\left.\right|_{\mathrm{OLi}} ^{\mathrm{C}}-\mathrm{Bu} \stackrel{\mathrm{H}_{2} \mathrm{O}}{\longrightarrow} \mathrm{CB}-\left.\right|_{\mathrm{OH}} ^{\mathrm{C}_{\mathrm{O}}-\mathrm{Bu}} \\
& \mathrm{CB}>\mathrm{C}=\mathrm{O} \stackrel{\mathrm{BuLi}}{\longrightarrow} \mathrm{CB}_{2}>\mathrm{C}_{\mathrm{Bu}}^{\prime} \stackrel{\mathrm{OLi}}{\mathrm{H}_{2} \mathrm{O}} \longrightarrow \mathrm{CB}>\mathrm{C}_{\backslash_{\mathrm{Bu}}^{\prime}}^{\prime O H}
\end{aligned}
$$

\section{Initiating Activity of Azo Groups Introduced onto Carbon Black}

The polymerization of MMA was carried out in the presence of carbon black 2 at $70^{\circ} \mathrm{C}$ and the initiating activity of the azo groups on the surface was examined. The results are summar- 
Table II. Initiating activity of azo groups introduced onto carbon black surface ${ }^{\mathbf{a}}$

\begin{tabular}{lccc}
\hline \multirow{2}{*}{ Carbon black } & Conversion & & Grafting \\
\cline { 2 - 2 } & $\%$ & $\%$ \\
\hline None & 0.7 & - \\
Untreated & 0 & & - \\
ACV-adsorbed & 0 & & - \\
2 & 4.6 & & 40.0 \\
\hline
\end{tabular}

a Carbon black, $0.30 \mathrm{~g} ; \mathrm{MMA}, 10.0 \mathrm{ml} ; 70^{\circ} \mathrm{C} ; 6 \mathrm{~h}$.

ized in Table II.

As shown in Table II, the thermal polymerization of MMA proceeded slightly even in the absence of initiator, whereas the rate of polymerization was negligibly small. In the presence of untreated carbon black, however, no polymerization of MMA was observed at all. This phenomenon suggests that in the presence of untreated carbon black, the thermal polymerization is completely inhibited by oxygen containing groups on the carbon black such as phenolic hydroxyl and quinonic oxygen groups. On the contrary, the polymerization of MMA was found to be initiated in the presence of carbon black 2 and poly(methyl methacrylate) (PMMA) was grafted onto the surface.

To ensure initiation by the azo groups on the surface, the initiating activity of ACVadsorbed carbon black was evaluated. ACVadsorbed carbon black was prepared by the treatment of untreated carbon black with ACV in a manner similar to the introduction of azo groups. As shown in Table II, ACV-adsorbed carbon black had no ability to initiate the polymerization. This suggests that ACV adsorbed on the carbon black surface during the treatment for the introduction of azo groups is completely removed by washing with methanol after the treatment.

Based on the above results, it is concluded that the polymerization of MMA is initiated by azo groups introduced onto the surface of the carbon black.

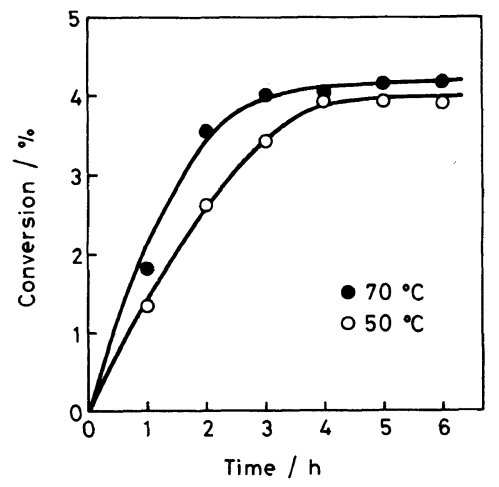

Figure 1. Graft polymerization of MMA initiated by carbon black 1. Carbon black, $0.30 \mathrm{~g}$; MMA, $10.0 \mathrm{ml}$.

\section{Graft Polymerization of MMA Initiated by Carbon Black 1}

The graft polymerization of MMA onto carbon black was examined by use of carbon black 1 as an initiator. Figure 1 shows the time-conversion curves of the polymerization of MMA at 50 and $70^{\circ} \mathrm{C}$.

As seen in Figure 1, it is apparent that carbon black 1 also has an ability to initiate the polymerization of MMA. The polymerization was of dead end type and the conversion did not increase even if the polymerization time was extended to $6 \mathrm{~h}$. This may be due to low content of azo groups.

The carbon black obtained from the polymerization produced a stable colloidal dispersion in a good solvent for PMMA: the precipitation of the carbon black particles from the dispersion of the carbon black obtained from the polymerization was scarcely observed even after 30 days. This suggests that PMMA is grafted from the carbon black surface. Therefore, the percentage of grafting was determined.

Figure 2 shows the relationship between the polymerization time and percentage of grafting using carbon black 1 as an initiator. The percentage of grafting increased to $40 \%$ as the polymerization proceeded. On the other hand, grafting efficiency (the proportion of grafted polymer to total polymer formed) at $50^{\circ} \mathrm{C}$ and 


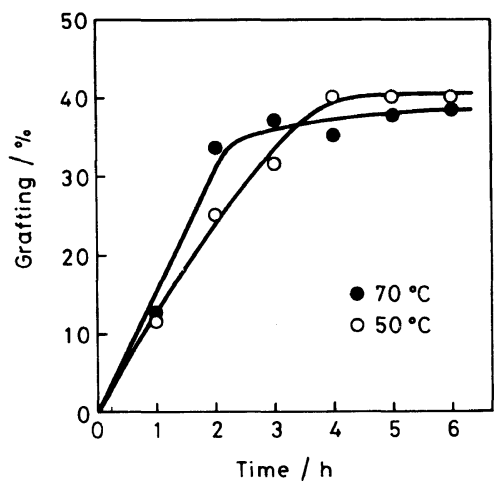

Figure 2. Relationship between polymerization time and percentage of grafting using carbon black 1 as an initiator.

$70^{\circ} \mathrm{C}$ was $44-45 \%$ and $38-50 \%$, respectively. Although PMMA was grafted onto the carbon black during the polymerization of MMA initiated by $2,2^{\prime}$-azobisisobutyronitrile (AIBN) in the presence of untreated carbon black, the percentages of grafting and grafting efficiency were less than $10 \%$ and $1.0 \%$, respectively. Therefore, the percentages of grafting and grafting efficiency in the polymerization initiated by azo groups on the surface were much larger than those obtained from the polymerization in the presence of untreated carbon black using AIBN as an initiator. This may be due to that PMMA is propagated from the radical on the surface produced by the decomposition of the azo groups (eq 1). The formation of ungrafted PMMA was considered to be based on the propagation from another initiator fragment (eq 1).

\section{Graft Polymerization of MMA Initiated by Carbon Black 2}

As shown in Table $\mathrm{I}$, the azo group content of carbon black 2 was larger than that of carbon black 1. Therefore, the initiating activity of carbon black 2 for the graft polymerization of MMA was examined. The results are shown in. Figures 3 and 4.

Figures 3 and 4 show that carbon black 2 also has an ability to initiate the polymerization of MMA. The conversion and percentage of

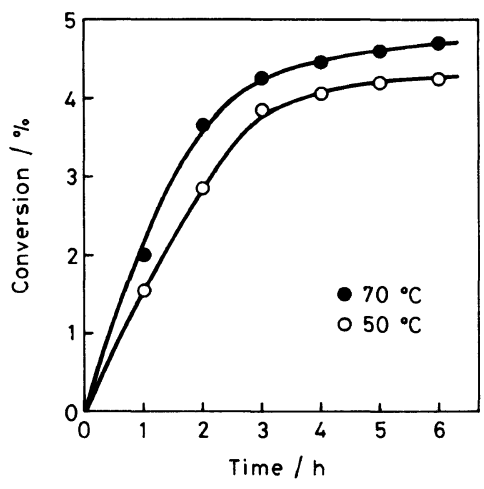

Figure 3. Graft polymerization of MMA initiated by carbon black 2. Polymerization conditions are given in Figure 1.

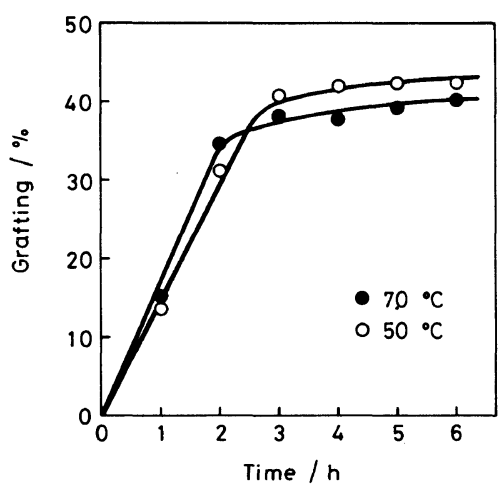

Figure 4. Relationship between polymerization time and percentage of grafting using carbon black 2 as an initiator.

grafting after $6 \mathrm{~h}$ in the presence of carbon black 2 are slightly larger than those of carbon black 1. In comparison with carbon black 1 , the initiating activity of carbon black 2 and the percentage of grafting, however, are not so large as expected.

The grafting efficiency at $50^{\circ} \mathrm{C}$ and $70^{\circ} \mathrm{C}$ in the graft polymerization was determined to be $45-58 \%$ and $40-50 \%$, respectively. These values are almost equal to those initiated by carbon black 1.

Graft Polymerization of MMA Initiated by Carbon Black 3

Figures 5 and 6 show the results of graft polymerization of MMA initiated by carbon 


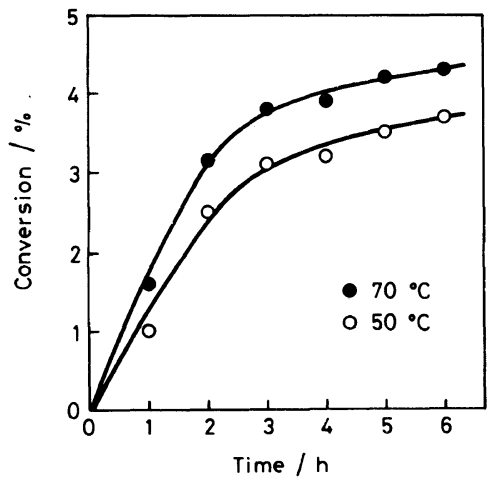

Figure 5. Graft polymerization of MMA initiated by carbon black 3. Polymerization conditions are given in Figure 1.

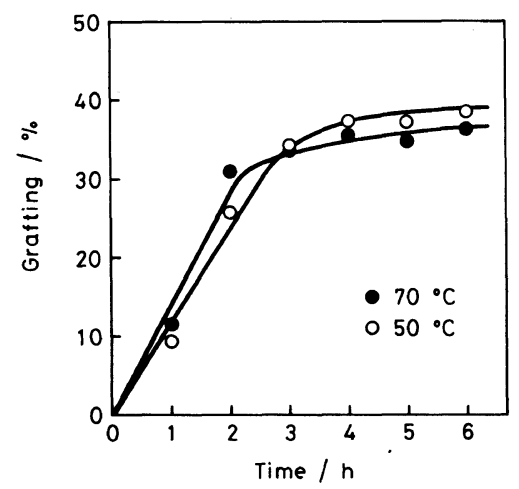

Figure 6. Relationship between polymerization time and percentage of grafting using carbon black $\mathbf{3}$ as an initiator.

black 3. From these results, it is concluded that the graft polymerization of MMA is initiated by carbon black 3 to give PMMA-grafted carbon black. The initiating activity and percentage of grafting, however, were lower than those of carbon black 1 or 2 , because of the lowest content of azo groups.

\section{Graft Polymerization of MMA Initiated by Carbon Black 4}

As shown in Table $\mathrm{I}$, by the reaction of the BuLi-treated carbon black with ACV-COCl, carbon black 4 with the highest azo group content was obtained: its azo group content increased to $0.48 \mathrm{mmol} \mathrm{g}^{-1}$. Figure 7 shows the time-conversion curves of the polymerization

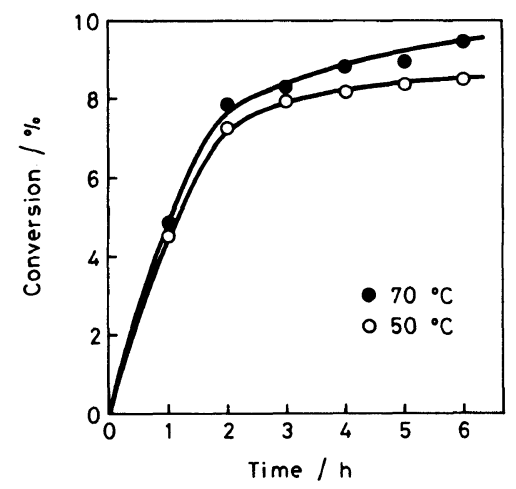

Figure 7. Graft polymerization of MMA initiated by carbon black 4. Polymerization conditions are given in Figure 1.

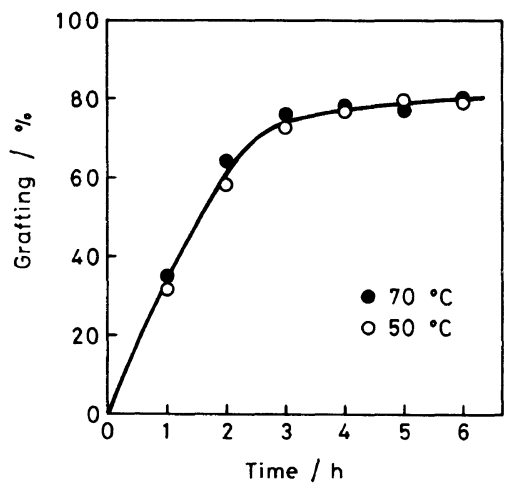

Figure 8. Relationship between polymerization time and percentage of grafting using carbon black 4 as an initiator.

of MMA initiated by carbon black 4 .

As shown in Figure 7 , the rate of the polymerization and conversion after $6 \mathrm{~h}$ were found to increase remarkably compared with those of carbon black 1, 2, or 3 .

Figure 8 shows the relationship between the percentage of grafting and the polymerization time initiated by carbon black 4 . The percentage of grafting increased to $80 \%$ as the polymerization proceeded.

Initiating Activity of Carbon Black 1, 2, 3, and 4

The initiating activities of carbon black 1, 2 , 3 , and 4 were summarized in Table III. It was found by comparing carbon black 3 and 4 that 
the initiating activity and the percentage of grafting increase with an increase of azo group content of carbon black. On the other hand, by comparing carbon black 1 and 3 , whose azo group content is almost equal, the initiating activity and the percentage of grafting were found to be almost equal. This suggests that the difference of half-life for decomposition between ACP and ACV is decreased by the bonding onto the carbon black surface. This may be due to that both ACP and ACV are bonded onto the carbon black surface with ester bond. Further detailed investigation, however, is required to estimate the relationship between the structure of azo groups introduced onto the surface and initiating activity.

Table III. Relationship between initiating activity of the polymerization and azo group content of carbon black

\begin{tabular}{|c|c|c|c|}
\hline \multirow{2}{*}{ Carbon black } & Azo group & Conversion & Grafting \\
\hline & $\mathrm{mmolg}^{-1}$ & $\%$ & $\%$ \\
\hline 3 & 0.13 & 3.8 & 35.9 \\
\hline 1 & 0.18 & 4.1 & 38.5 \\
\hline 2 & 0.33 & 4.6 & 40.0 \\
\hline 4 & 0.48 & 9.5 & 80.0 \\
\hline
\end{tabular}

a Polymerization conditions are given in Table II.

\section{Graft Polymerization of Various Vinyl Mono- mers}

The surface active radicals on carbon black, which are formed by the reaction of initiator fragments with carbon black, readily capture the growing polymer radicals with negative $e$-value, but not with positive $e$-value. ${ }^{16-18}$ Therefore, the polymerization of vinyl monomers with negative $e$-value in the presence of carbon black using peroxides as initiator is markedly retarded, whereas that with positive $e$-value is scarcely retarded. This is due to that radicals formed by the decomposition of peroxides have ability to abstract hydrogen atoms from carbon black to give surface active radicals. On the contrary, when azo initiators are used, remarkable retardation or inhibition by carbon black is scarcely observed regardless of $e$-value of vinyl monomers, because azo initiators have no ability to produce surface active radicals. ${ }^{16-18}$

In the previous paper, ${ }^{9,19}$ we reported that the polymerization of vinyl monomers with positive $e$-value was initiated by peroxyester groups introduced onto the carbon black surface. Peroxyester groups on carbon black, however, failed to initiate the graft polymerization of vinyl monomers with negative $e$-value. Therefore, it is impossible to graft vinyl

Table IV. Effect of $e$-value of vinyl monomers on the graft polymerization using azo groups introduced onto carbon black surface ${ }^{a}$

\begin{tabular}{|c|c|c|c|}
\hline \multirow{2}{*}{ Monomer } & \multirow{2}{*}{$e$-value } & \multirow{2}{*}{ 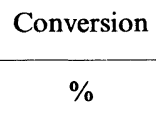 } & \multirow{2}{*}{$\begin{array}{c}\text { Grafting } \\
\%\end{array}$} \\
\hline & & & \\
\hline$N$-Vinyl-2-pyrrolidone & -1.14 & 2.6 & 74.3 \\
\hline Styrene & -0.80 & 2.0 & 28.5 \\
\hline 4-Vinylpyridine & -0.28 & 10.3 & 79.9 \\
\hline Vinyl acetate & -0.22 & 2.3 & 20.5 \\
\hline 2-Hydroxyethyl methacrylate & 0.20 & 1.7 & 24.0 \\
\hline Methyl methacrylate & 0.40 & 4.7 & 40.0 \\
\hline Glycidyl methacrylate & 0.57 & 10.6 & 87.5 \\
\hline Methyl vinyl ketone & 0.68 & 4.6 & 35.2 \\
\hline Acrylic acid & 0.77 & 6.2 & 64.8 \\
\hline Acrylonitrile & 1.20 & 13.1 & 76.2 \\
\hline
\end{tabular}

a Carbon black $2,0.30 \mathrm{~g}$; monomer, $10.0 \mathrm{ml} ; 70^{\circ} \mathrm{C}$. 
polymers such as polystyrene and poly(vinyl acetate) by use of peroxyester groups on the carbon black surface.

Therefore, the effect of $e$-value of vinyl monomers on the graft polymerization initiated by azo groups on the carbon black surface was investigated. The results are shown in Table IV. As shown in Table IV, it is apparent that carbon black 2 has an ability to initiate the graft polymerization of vinyl monomers regardless of the $e$-value of monomers, and the corresponding polymers are effectively grafted onto the surface. These results indicate that the radicals formed by the decomposition of peroxyester groups on the carbon black produce surface active radicals by hydrogen abstraction, whereas that of azo groups on the carbon black produce no surface active radicals.

Accordingly, it is concluded that the graft polymerization of various vinyl monomers is initiated by azo groups introduced onto the carbon black surface to give polymer-grafted carbon black with a higher percentage of grafting.

\section{REFERENCES}

1. R. Kroker, M. Schneider, and K. Hamann, Prog. Org. Coatings, 1, 23 (1972).
2. R. Laible and K. Hamann, Adv. Colloid Interface Sci., 13, 65 (1980).

3. N. Tsubokawa, Nippon Gomu Kyokaishi, 58, 306 (1985).

4. N. Tsubokawa and T. Endo, Kinou Zairyoh, 8, 13 (1988); Tanso, No. 140, 322 (1989).

5. N. Tsubokawa, A. Funaki, Y. Hada, and Y. Sone, J. Polym. Sci., Polym. Chem. Ed., 20, 3297 (1982).

6. N. Tsubokawa, T. Ohyama, A. Yamada, and Y. Sone, J. Polym. Sci., Polym. Chem. Ed., 23, 489 (1985).

7. N. Tsubokawa, J. Polym. Sci., Polym. Chem. Ed., 22, 1515 (1984); ibid., 25, 1979 (1987).

8. N. Tsubokawa, K. Fujiki, and Y. Sone, J. Macromol. Sci.-Chem., A25, 1159 (1988).

9. N. Tsubokawa, K. Fujiki, and Y. Sone, Polym. J., 20, 213 (1988).

10. A. Ueda, Y. Shiozu, Y. Hidaka, and S. Nagai, Kobunshi Ronbunshu, 33, 131 (1976).

11. N. Tsubokawa, K. Kobayashi, and Y. Sone, Polym. Bull., 17, 87 (1987).

12. E. Papirer, V. T. Nguyen, and J. B. Donnet, Carbon, 16, 141 (1978).

13. R. Yosomiya, T. Fujisawa, and K. Morimoto, Polym. Bull., 12, 523 (1984).

14. R. Yosomiya, K. Morimoto, and T. Suzuki, J. Appl. Polym. Sci., 29, 671 (1984).

15. W. I. Bengough and H. W. Melville, Proc. R. Soc. (London), Ser. A, 249, 445 (1959).

16. K. Ohkita, N. Tsubokawa, E. Saitoh, M. Noda, and N. Takashina, Carbon, 13, 443 (1975).

17. K. Ohkita, N. Tsubokawa, and E. Saitoh, Carbon, 16; 41 (1978).

18. K. Ohkita, N. Tsubokawa, and N. Takashina, Nippon Gomu Kyokaishi, 49, 223 (1976).

19. K. Fujiki, N. Tsubokawa, and Y. Sone, Tanso, No. 140, 234 (1989). 